\title{
Sphenoid sinus fungall ball: a retrospective study over a 10- year period*
}

\author{
Ph. Eloy' ${ }^{1}$ J. Grenier², A. Pirlet' ${ }^{1}$, A.L. Poirrier'1, J.S. Stephens³, Ph. Rombaux² \\ ' ENT Department CHU UCL Mont-Godinne 5530 Yvoir, Belgium \\ 2ENT Department Cliniques Universitaires Saint-Luc Avenue Hippocrate, 101200 Bruxelles, Belgium \\ ${ }^{3}$ ENT department Royal National Throat Nose \& Ear Hospital 330 Gray's Inn Road London, WC1X 8DA, United Kingdom
}

\author{
Rhinology 51: 181-188, 2013 \\ DOI:10.4193/Rhino12.114 \\ *Received for publication: \\ July 2, 2012
}

Accepted: February 7, 2013

\section{Summary}

A fungal ball consists of a dense conglomerate of fungal hyphae growing at the surface of the sinus mucosa without tissue infiltration. The maxillary sinus is by far the most commonly involved paranasal sinus cavity followed by the sphenoid sinus. The present study is a retrospective study of 25 consecutive cases treated during the last 10 years in the two hospitals belonging to the Catholic University of Louvain (CHU Mont-Godinne and UCL Saint Luc). We report the symptomatology, the imaging and discuss the different surgical managements.

We conclude that the clinician must have a high index of suspicion when dealing with a unilateral rhinosinusitis persisting despite a maximal and well conducted medical treatment. This is particularly so in elderly women when associated with facial pain and post nasal drip, particularly when the computed tomography shows an unilateral opacity of the sphenoid sinus with or without a sclerosis or an erosion of the bony walls, a polyp in the sphenoethmoidal recess or a hyperdensity mimicking a foreign body.

An endonasal endoscopic sphenoidotomy is the treatment of choice in most cases, allowing good ventilation of the sinus and radical removal of all the fungal concretion. A biopsy of the sinus mucosa adjacent to fungal elements is of upmost important to confirm the non- invasiveness of the fungi within the tissue. Antifungal medication is not required in uncomplicated forms. All host factors producing some degree of immunosuppression must be corrected when present and must alert the clinician to rule out any forms of invasive disease.

Key words: sphenoid sinus, sinus fungus ball, surgical approaches, retrospective study

\section{Introduction}

Fungi are ubiquitous organisms. They are present in the soil, decaying materials and in the air. The concentrations of their spores depend on environmental conditions such as the place, climate and seasons ${ }^{(1,2)}$. They can cause respiratory diseases in the nose, paranasal sinus cavities, bronchi and lungs. They can colonize and infect the upper airways by two ways: the aerogenic pathway (inhalation of the spores) and the odontogenic pathway.

In case of a maxillary fungal sinusitis, it is essential to rule out any dental problem. Indeed, overfilling with a zinc-oxide-eu- genol dental paste during an endodontic care on an upper jaw tooth is a major factor for the development of a sinus problem ${ }^{3-}$ 9). At the beginning the material penetrates into the sinus cavity and plays an antibacterial role, but with time, fungi can develop. The clinical presentation depends on the location of the fungal disease, the fungal species, the immune status and the mucosal or tissue condition ${ }^{(1-9)}$.

Five basic diagnostic categories of fungal paranasal sinus disorders are currently recognized ${ }^{(10-13)}$. They are classified as invasive or non-invasive forms based on histopathological findings. 
Three types are true tissue-invasive infectious diseases: acute fulminant necrotizing fungal rhinosinusitis, chronic invasive fungal rhinosinusitis and granulomatous invasive fungal rhinosinusitis caused mainly by Aspergillus flavus. They mostly affect the immunocompromised patients and can be life-threatening systemic illness. On histology, fungal elements are visualized microscopically on Gomori methenamine silver or other special stains for fungus within the tissue, the sinus mucosa, but also the blood vessels and the underlying bone.

In non-immunocompromised patients, the fungal disease is non-invasive and extramucosal ${ }^{(14)}$. The fungal elements proliferate at the surface of the sinus mucosa but not in its depth; the diagnosis is established by the pathologist who identifies the fungal hyphae in the sinus contents (secretions or concretions). The diagnosis of the fungal species depends on mycological workup but a positive culture is reported in only 10.5 to $72.7 \%$ of the cases in the literature ${ }^{(15,16)}$.

The non-invasive group contains 2 different forms of extramucosal fungal rhinosinusitis (the sinus fungal ball and the allergic fungal rhinosinusitis.

SFB (sinus fungus ball) is the most common form of extramucosal fungal rhinosinusitis. Colonization is mainly caused by Aspergillus species in Europe and particularly Aspergillus fumigatus. It usually involves the maxillary or sphenoid sinus. It is characterized by a dense and compact conglomerate of fungal hyphae separate from but adjacent to sinus respiratory mucosa ${ }^{(17)}$. In the surrounding sinus mucosa there is a chronic inflammatory reaction of variable intensity. This response includes lymphocytes, plasma cells, mast cells and eosinophils without an eosinophilic predominance or a granulomatous response. Allergic mucin is absent on haematoxylin-eosin stained material. Table 1 demonstrates the criteria for the diagnosis of a sinus

Table 1. Adapted from de Shazo R. ${ }^{(17)}$ : Criteria for the diagnosis of a mycetoma.

1. Radiologic evidence of sinus opacification with or without associated flocculent calcifications

2. Mucopurulent, cheesy, or clay-like greasy material within a sinus

3. A matted, dense conglomeration of hyphae separate from but adjacent to sinus respiratory mucosa

4. A chronic inflammatory response of variable intensity in the mucosa adjacent to fungal elements. This response includes lymphocytes, plasma cells, mast cells, and eosinophils without an eosinophil predominance or a granulomatous response. Allergic mucin is absent on hematoxylin-eosin-stained material

5. No histologic evidence of fungal invasion of mucosa, associated blood vessels, or underlying bone visualized microscopically on Gomori methenamine silver or other special stains for fungus. fungus ball ${ }^{(17)}$.

Allergic fungal sinusitis (AFS) is much more common in the United States of America, India and South East countries. The hot and dry tropical climate is a great contributing factor.

The disease was first described by Katzenstein in $1983^{(18)}$. The histological pattern is similar to that observed in case of Allergic Bronchopulmonary Aspergillosis. There is a very thick, tenacious and clay-like mucus; in this mucus are many layers of eosinophils in varying states of preservation and Charcot-Leyden crystals, resulting from the degradadation of the eosinophils ${ }^{(19)}$. In this mucus some scanty and scattered fragmented fungal hyphae can be visualized. Mycological studies have revealed that Aspergillus species count only for $15 \%$ of the cases. In the remaining $85 \%$, we find black Aspergillus-like fungal species (dematiaceous), such as Bipolaris, Dreschlerae or Curvularia ${ }^{(20)}$, hence it is more appropriate to call this entity "allergic fungal sinusitis" rather than "Allergic Aspergillus sinusitis" as proposed initially by Katzenstein. Moreover, as atopy is not frequently encountered, the right terminology should be "eosinophilic fungal rhinosinusitis" (21,22).

In this paper, we report a cohort of 25 patients presenting with a sphenoid sinus fungal ball (SSFB). We review the clinical features, the demography, the favorizing factors, the imaging and the modalities of treatment.

\section{Material and method}

This is a retrospective study conducted in the 2 hospitals ( $C H U$ Mont-Godinne and UCL Saint Luc) belonging to the Catholic University of Louvain. We reviewed the files of patients treated for a sphenoid sinus fungal ball (SSFB) during the last 10 years. A CT scan was performed in all the patients. In case of doubt, a MRI was ordered. Surgery was performed exclusively endonasally. A biopsy of the sinus mucosa adjacent to the fungus ball was performed in all the cases. The diagnosis of SSFB was established by the pathologist. Mycological studies were performed in 18 out of the 25 patients.

The follow-up consisted of a nasal endoscopy in all cases and a sinus CT scan in case of recurrence or persistence of the symptomatology or to document the postoperative result.

\section{Results}

This study includes 25 patients: 17 women and 8 men. The sex ratio $\mathrm{F} / \mathrm{M}$ ) was : $2 / 1$. The average age was 62 years old (range : 24- 83). Twenty-one patients were immunocompetent whereas 4 patients had host factors predisposing to invasive fungal infections. One patient had type 1 diabetes mellitus, another had type 2 diabetes mellitus, another regularly used systemic glucocorticoids for lung disease (corticodependent asthma) and the 4 th patient received hydroxycarbamide $\left(\mathrm{Hydrea}^{\circledR}\right)$ for 
Table 2. Radiological findings.

\begin{tabular}{lc} 
Radiological findings & Number of patients \\
\hline Unilateral disease & 5 \\
Bilateral disease & 11 \\
Complete opacity of the sphenoid sinus & 14 \\
Partial opacity within the sphenoid sinus & 18 \\
Sclerosis of the sphenoid walls & 10 \\
Microcalcifications & 9 \\
Polyp in the sphenoethmoidal recess & 3 \\
Pseudotumoral aspect - Bone erosion &
\end{tabular}

chronic myeloid leukemia.

Concerning the symptomatology, 15 patients suffered from pain. The topography was mostly retro- or periorbital, but the patients may also complain of facial pain, otalgia or pain in the neck. Thirteen reported post nasal dripping. A proportion of patients complained of nasal obstruction, hyposmia or coughing. One patient presented with a decrease in his visual acuity and 4 patients had diplopia due to a 6th cranial nerve palsy. No patients experienced any intracranial complications (stroke, meningitis, brain abscess) nor blindness.

On imaging, the disease was unilateral in 20 cases and bilateral in 5 cases. Eleven patients had a complete sinus opacity whereas the remaining cases had a partial opacity. A polyp in the sphenoethmoidal recess was visualized in 9 patients. Microcalcifications in the sphenoid sinus were visualized in 10 patients. $A$ sclerosis of the bony walls of the sphenoid sinus was noted in 18 patients. The sinus cavity was expanding with bone erosion in 4 patients. One patient had a sphenoid sinus mucocoele associated with the SSFB.

Figure 1 illustrates the different presentations of the SSFB on a CT scan and Table 2 reports the different radiological findings in our patients. Figure 2 illustrates the correlation between the CT and the MRI and Figure 3 the correlation between the CT scan and the intraoperative nasal endoscopy.

The management was surgical in all the cases. In 16 patients it consisted of an unilateral endonasal endoscopic sphenoidotomy performed through the sphenoethmoidal recess. In 4 patients a complete ethmoidectomy was carried out in addition to the sphenoidotomy. In one patient the posterior ethmoid was opened. In 4 patients the surgery consisted of a a paraseptal approach with resection of the posterior nasal septum and drilling of the anterior walls of both sphenoid sinuses. In 3 out of these 4 cases the patients had undergone a complete ethmoidectomy in the past. The mean postoperative follow-up was 24 months (range: 6 to 120 months)

Two patients had a revision surgery. In one case for recurrence of the fungal disease; in the other case no fungus was discovered at surgery - rather a fibrotic web had closed the neo-ostium. Both of these patients had previously undergone a unilateral sphenoidotomy. In each case, revision revision surgery comprised a paraseptal approach.

Figure 4 illustrates the different endonasal approaches to the sphenoid sinus.

The definitive diagnosis was always established by the pathologist. There were no cases of invasive fungal rhinosinusitis. Aspergillus fumigatus grew in 6 out of 18 cases. In 3 patients Aspergillus species was found at culture of secretions from endobronchial aspiration.

\section{Discussion}

Fungal rhinosinusitis is relatively common in Europe ${ }^{(16,23,24)}$ and probably underdiagnosed because of its nonspecific symptoms and the presence of asymptomatic forms in 10 to $20 \%$ of the cases ${ }^{(15,16,24)}$. Nevertheless, the incidence has increased dramatically over the last two decades. In two respective studies on patients undergoing maxillary sinus surgery, the prevalence of SFB was $13 \%$ and $28.5 \%$, respectively ${ }^{(25,26)}$. Aspergillus species are the major causative agents and the maxillary antrum the most frequently involved paranasal sinus cavity. The development of a fungal maxillary rhinosinusitis after an endodontic care on an upper jaw tooth is very frequent with an incidence estimated as high as $84 \%$ of the cases in some publications ${ }^{(4-9,13,15,16,23,24,26)}$. The clinician must have a high index of suspicion when a unilateral rhinosinusitis persists despite a maximal and well-conducted medical treatment with or without cacosmia or dental pain. For the sphenoid sinus the story is a little bite different. Isolated sphenoid disease accounts for $2.7 \%$ of all sinus infections according to Lew et al. ${ }^{(27)}$ and less than $1 \%$ according to Hnatuk et al (28). The incidence of fungal sphenoiditis amongst patients with isolated sphenoid disease varies from 4.5 to $26.8 \%{ }^{(29-32)}$. This easily explains the limited number of patients $(n=25)$ included in this series over a 10-year period but also in the series published by Bowman $(n=17)^{(33)}$, Dufour $(n=20)^{(23)}$, Ferreiro $(n=10)^{(34)}$, Herman $(n=24)^{(35)}$, Lee $(n=12)^{(36)}$, Klossek $(n=10)^{(37)}$, Pagella ( $\mathrm{n}=20)^{(15)}$, Nicolai $(\mathrm{n}=23)^{(24)}$ and Thery $(\mathrm{n}=15)^{(38)}$.

The disease more frequently affects elderly patients (mean age 60 years old) and particularly women ${ }^{(33-38)}$ with a female to male ratio of 2:1 in this series. The disease is usually confined to the 
sphenoid sinus.

The pathogenesis of SSFB is still a subject of debate. The odontogenic route of contamination of the sphenoid sinus seems to be excluded. The aerogenic route of contamination is most likely. Some patients may also have host factors predisposing to invasive fungal infection. Two patients had type 1 or type 2 diabetes mellitus. Prolonged systemic corticosteroid therapy was observed in 1 patient. These associations have already be reported in the literature, respectively ${ }^{(25,33,38)}$. Another patient received a cytostatic agent (hydroxycarbamide $\left(\right.$ Hydrea $^{\circledR}$ ) for a chronic myeloid leukemia. In all these cases the pathologist did not visualize any fungal tissue invasion.

The symptomatology can be acute or chronic ${ }^{(35)}$. In most of our patients, the symptoms had been present for more than 3 months. Facial pain and post nasal drip are the two most commonly reported complaints ${ }^{(33-39)}$. The facial pain was retro- or periorbital. They can be explained by the pressure into the sphenoid cavity when the sinus was completely filled by the concretions, or by the irritation (inflammation) of the dura covering the planum sphenoidale and the anterior cranial fossa in case of a pseudotumoral aspect, or by the irritation of the ophthalmic division of the trigeminal nerve that supplies the sphenoid sinus and provides innervations to much of the head. Post nasal drip is very common and associated with cough. The secretions can be mucoid, purulent or blood stained. This symptom is quite common, present in $52 \%$ of the cases but it is not specific to a SSFB, as it can be present in case of a chronic maxillary or ethmoidal rhinosinusitis.

Although most of the cases were uncomplicated in this series, SSFB can be associated with orbital ( blindness, reduction of the vision and diplopia) or intracranial (death, meningitis, brain abscess) complications $(25,33,35,38,40,41,42)$.

One of our patients suffered from a reduction of his visual acuity and 4 patients presented with diplopia. In one case, the fungal disease was associated to a sphenoid mucocele. In the other cases, the "sinusitis" resulted in a pseudotumour with expansion and destruction of the sinus walls. The diplopia could occur as a result of compression of the abducens nerve (Vlth cranial nerve), the longest nerve running in the lateral wall of the sphenoid sinus within the medial portion of cavernous sinus. In all cases, the reduced vision and diplopia resolved completely after surgery. In no cases was the sinusitis invasive. We did not give any antifungal medication and did not observe any other complications.

Endoscopic examination failed to identify abnormalities in most patients. Polyps in the sphenoethmoidal recess were observed in only 9 out of 25 patients.
Because the symptomatology was vague and unspecific except in case of visual disturbance or diplopia, an imaging was mandatory to establish the diagnosis particularly in cases of headache (25,38,43-45).

CT can be very specific by showing partial or complete heterogeneous sinus opacity associated to microcalcifications. In our series, on the $\mathrm{CT}$, the pathology was mostly unilateral $(\mathrm{n}=$ $20 / 25)$ and consisted with a partial (14/25) or complete (11/25) opacity of the sphenoid sinus. The second radiological finding was a sclerosis and thickening of the bony confines of the sinus, present in 14 patients. This can result from an osteitis of the sphenoid walls. This finding usually persists after successful surgery ${ }^{(33)}$. Microcalcifications are present in less than half of the patients ( $n=10$ out of 25 ). This is clearly less common than in cases of a maxillary fungal ball whereas microcalcifications are present in 60 to $80 \%$ of the cases ${ }^{(23,24,37,38,43-45)}$. A polyp in the sphenoethmoidal recess was present in only 9 out of 25 patients. In 4 cases, there were radiological signs of bone erosion. Although bone lysis is suggestive of invasive fungal infections, fungal tissue invasion was absent in all cases. This is a form of so-called chronic erosive or chronic non invasive destructive sinusitis ${ }^{(25,46-48)}$. This entity is characterized by a quite extensive disease and bone erosion on CT scan imaging without evidence of tissue invasion by fungi. Osteolysis is thought to be the consequence of chronic inflammation induced by fungal growth. Clinically this can be asymptomatic ${ }^{(25)}$ or associated with neurological or ophthalmological symptoms (diplopia or blindness). Erosions of the sphenoid bone resulted in exposure of important neurovascular structures, the internal carotid artery, the optic nerve, and branches of the trigeminal nerves. This represents a potential risk of complications during the surgery. When present these bony changes persist postoperatively.

When there is a high index of suspicion of a fungal disease with or without an intracranial or intraorbital invasion but the $\mathrm{CT}$ findings are not conclusive for fungal disease, an MRI may be of some help in the differential diagnosis. However, possible artefacts related to the heavy metal (iron, manganese) and calcium content within fungal hyphae can interfere with the diagnosis. Typically, MRI demonstrates an iso- or hypointensity on T1 weighted images and marked hypointensity on T2-weighted images. Figure 2 illustrates such findings. In T1 weighted image a fungal ball appears as a non-enhancing often heterogeneous nodular hyposignal. After contrast, the inflamed peripheral mucosa may enhance ${ }^{(1,24,32)}$. T2 shortening is commonly related to very dry, concretized secretions and/or the manganese and iron particles in fungal hyphae ${ }^{(33)}$. It can also be seen in other clinical situations such as very dry secretions, mucocoeles and intrasinus hemorrhages. Therefore the MRI findings need to be evaluated in conjunction with the CT scan. 

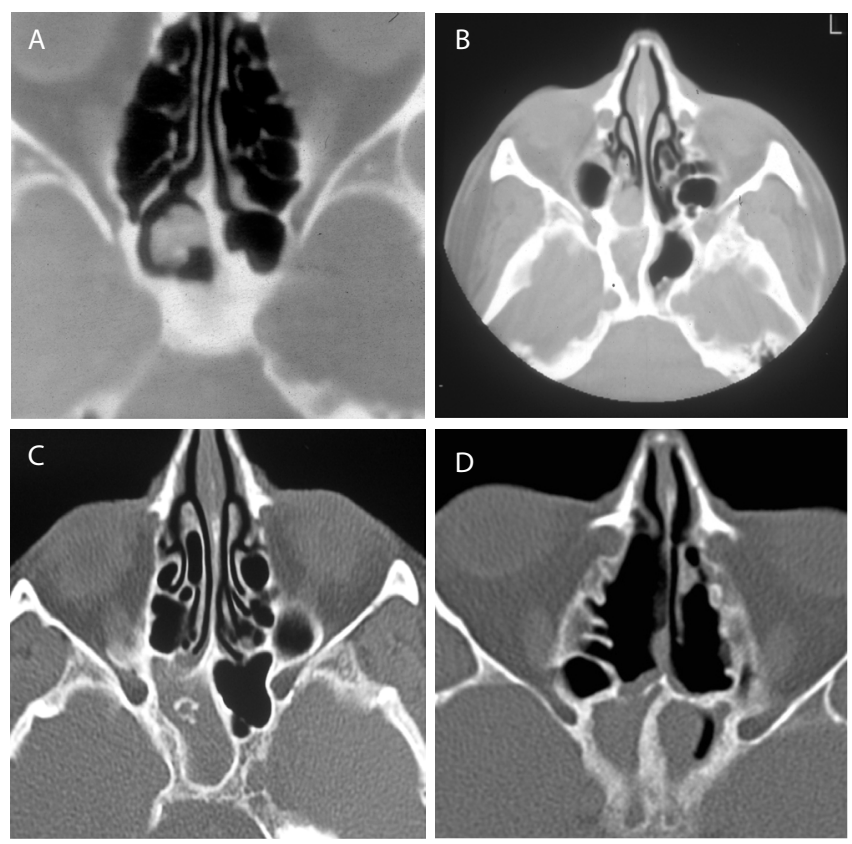

Figure1. Different presentations of a fungus ball in the sphenoid sinus on a CT.

A. Axial CT scanner - Polyp in the sphenoid sinus.

B. Axial CT scanner - Polyp in the sphenoethmoidal recess associated with a complete opacification of the sphenoid sinus and sclerosis of the bony walls.

C. Axial CT scanner - Microcalcification in the sphenoid sinus.

D. Axial CT scanner - Sclerosis of the sphenoid walls. Patient having had a bilateral ethmoidectomy in the past.

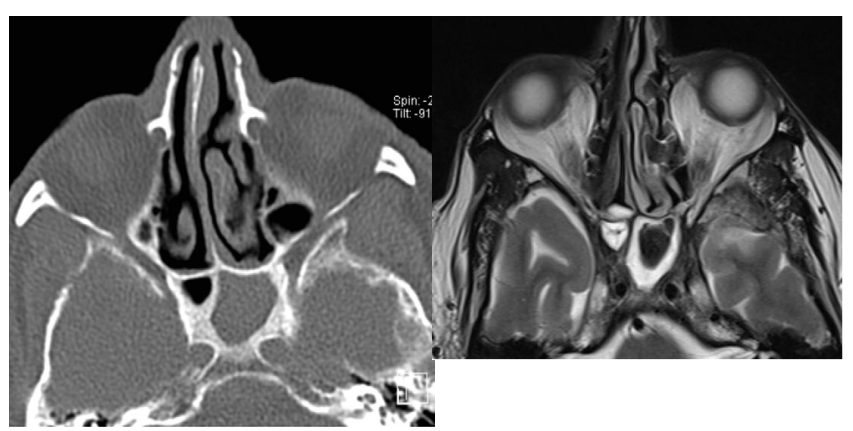

Figure 2. Comparison of a CT scanner and a MRI. The CT scan shows a unilateral and complete opacity of the left sphenoid sinus. On the MRI there is a hypointense signal on $\mathrm{T} 2$ weighted sequence and a enhancement of the sinus mucosa. These findings are compatible with the diagnosis of a sphenoid fungal disease.
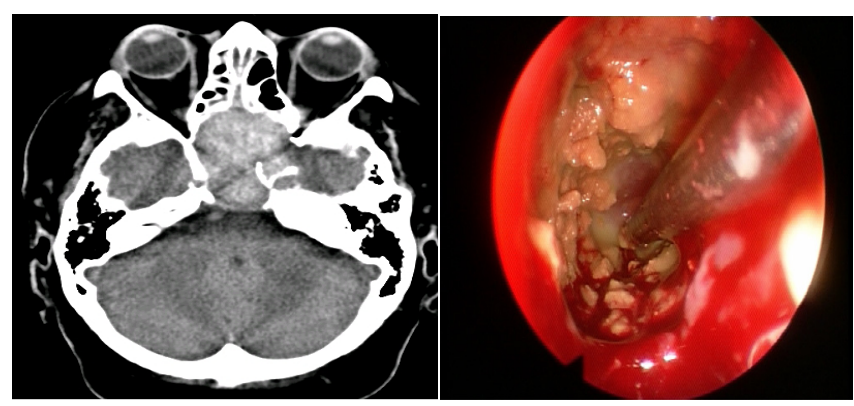

Figure 3. Comparison between a CT scan and the intraoperative view. The CT scan shows a pseudotumoral sinusitis and the endoscopic view confirms the presence of a grey material compatible with the diagnosis of a fungal sinusitis.
Exceptionally, 2 diseases can coexist, the fungal disease and a sphenoid sinus mucocoele, an association published by Lee in $2012^{(48)}$.

In case of "pseudotumoral appearance", an MRI is mandatory to rule out a tumour, but the diagnosis can also be approached endoscopically during the surgery. Figure 3 illustrates such a situation.

The diagnosis of SSFB is based upon the histopathological and mycological workup.

The definitive diagnosis of the fungus disease is established by the pathologist. Indeed the sensitivity and specificity of the histological examination are much higher than for the bacteriologic studies. In our series, all the cases of SSFB were diagnosed by the pathologist whereas Aspergillus grew on culture in only 6 out of 18 samples (30\%). This percent is reflected in the literature $(22-24,34,38,49)$. Fungal cultures do not contribute on a routine basis to establish the diagnosis because of this very low percentage of positive results. But, if we want to increase the sensitivity and specificity of the test, the specimen must be brought to the bacteriologist in a very short period of time, put in an optimal medium for culture after centrifugation and be examined directly and after a few days of incubation.

Moreover, it is worth to point out that if de Shazo ${ }^{(17)}$ proposes a list of criteria that enable the surgeon to evocate the diagnosis of a fungal disease, Broglie ${ }^{(49)}$ stresses on the fact that a severe inflammatory reaction of the surrounding tissue is commonly found in case of SFB and can therefore be used as an additional sign for fungal infection.

The treatment is surgical ${ }^{(27,32-39)}$. It consists of a radical removal of all the fungal concretion. In the majority of the cases it is possible to do so with a pure unilateral sphenoidotomy performed via the sphenoethmoidal recess ${ }^{(25,35,38)}$. To be sure that the removal of all the fungal debris is complete, it is useful to irrigate the sinus cavity with saline at high pressure as recommended by Nicolaï (24) except in case of bone erosion. Another interesting technical point is to clean the sinus cavity with a neurosurgical pledget soaked in a physiologic solution as proposed by Chao and Liu ${ }^{(50)}$. When the concretion is lateral and the sphenoid sinus well-pneumatized, we recommend a posterior ethmoidectomy in association with the sphenoidotomy using the $45^{\circ}$ or 

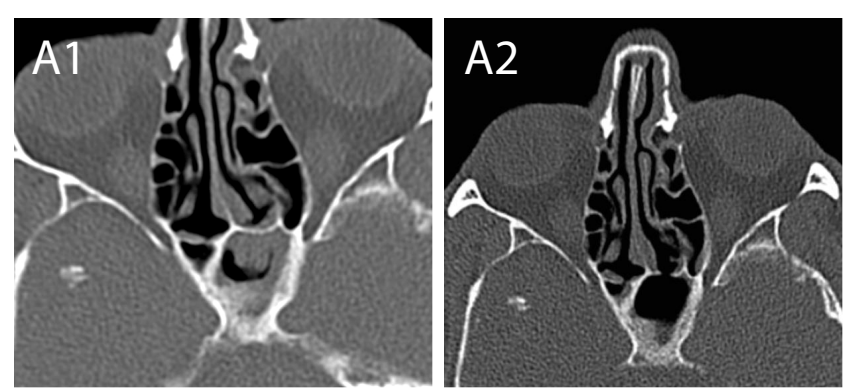

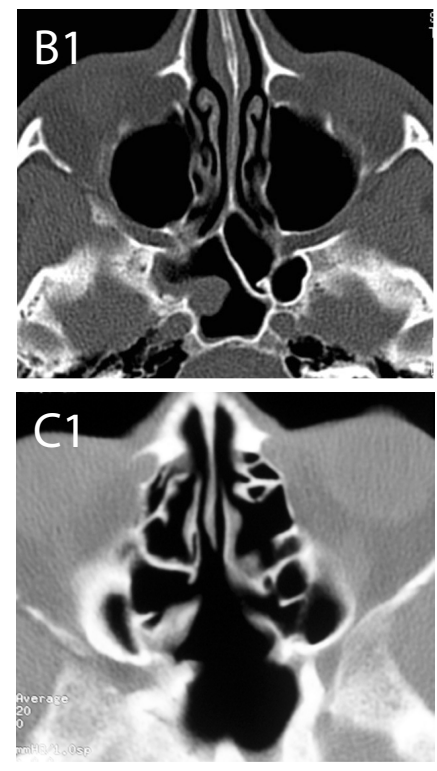

Pre-operation

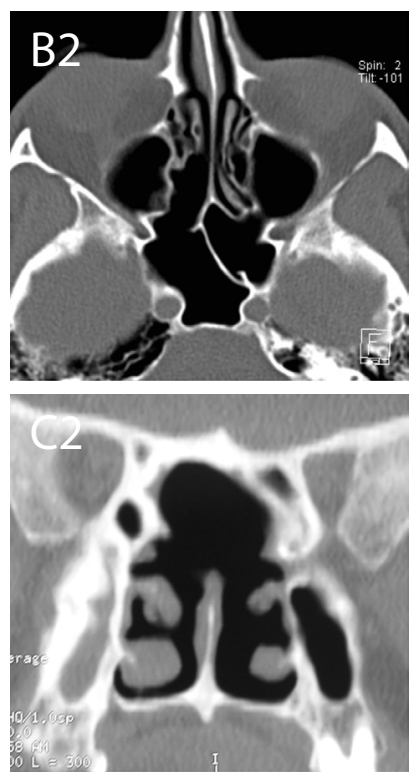

Post-operation
Figure 4. Illustration of the different types of endonasal surgery. A1-2. Illustration of a pure endonasal sphenoidotomy B1-2. Illustration of a posterior ethmoidectomy associated to the sphenoidotomy

C1-2. Illustration of a paraseptal approach: resection of the posterior septum and drilling of both anterior walls of the sphenoid sinuses.

the $70^{\circ}$ rigid telescope. This widens the approach and makes easier all manipulations in the sphenoid cavity. It is clear that no medical treatment can rescue an "incomplete" surgical resection.

Lastly, a paraseptal approach is recommended when the sphenoid sinuses are small and their walls very sclerotic, particularly in case of revision surgery. Actually, we know that in such a situation an isolated sphenoidotomy can close due a huge postoperative fibrosis. A paraseptal approach requires a drilling of the anterior walls of both sphenoid sinuses and a resection of the posterior septum close to the sphenoid rostrum. The different endonasal approaches are illustrated in Figure 4.
The surgery is usually associated with a high rate of success in the literature ${ }^{(24,34)}$. Recurrence is rare and can be explained by an incomplete removal of the fungal elements, particularly in highly pneumatized sphenoid sinus. More common is closure of the neoostium. This can be observed when the sphenoidotomy is too small, the sinus mucosa completely resected and the sphenoid bony walls very sclerotic. For this reason, we recommend in cases of revision surgery a paraseptal approach with resection of the posterior nasal septum and drilling of the anterior walls of the sphenoid sinuses.

During the surgery, it is mandatory to take biopsy of the mucosa adjacent to fungal ball for histopathologic determination of fungal invasiveness or not. A complete extirpation of the sinus mucosa is not necessary even when the mucosa is very hyperplastic $(16,24,25,29)$.

\section{Conclusion}

This study confirms that SSFB is an uncommon pathology involving mainly elderly women. We must consider the diagnosis when we deal with a rhinosinusitis resistant to medication associated to facial pain, postnasal drip and cough. The clinician should have a high index of suspicion when the CT scan shows a complete or a partial opacity of the sphenoid sinus associated to sclerosis, expansion or erosion of the bony walls, and/or a polyp in the sphenoethmoidal recess and/or microcalcifications. The treatment is surgical. In the majority of the cases a single pure endonasal sphenoidotomy is sufficient to achieve a complete surgical removal of the fungal concretion. A biopsy of the mucosa adjacent to the fungal elements is mandatory to confirm the diagnosis of extramucosal fungal rhinosinusitis. Antifungal agents are not recommended in uncomplicated cases but all host factors causing some degree of immunosuppression must be corrected. When present they should alert the clinician to the possibility of invasive fungal disease and to give a systemic antifungal medication postoperatively for a long period of time.

\section{Authorship contribution}

Pr Eloy was the main author, supervizes the study and takes the responsability of the contents of the manuscript.

The other coauthors have actively participated to the collect and review of the cases.

\section{Conflict of interest}

The authors do not have any conflicts of interest to declare. 


\section{References}

1. Grosjean P, Weber R. Fungus balls of the paranasal sinuses: a review. Eur Arch Otorhinolaryngol. 2007; 264: 461-470.

2. Hathiram BT, Khattar VS. Fungus balls of the paranasal sinuses. Otorhinolaryngol Clin. 2009; 1: 33-35.

3. Barry B, Topeza M, Gehanno P, Aspergillosis of the paranasal sinus and environmenta factors, Ann Otolaryngol Chir Cervicofac. 2002; 119: 170-173.

4. Dahl JE. Toxicity of endodontic filling Materials. Endodontic Topics 2005; 12 39-43.

5. Khongkhunthian $P$, Reichart PA Aspergillosis of the maxillary sinus as a complication of overfilling root canal material into the sinus: report of two cases. Endodontics 2001; 27: 476-478.

6. De Foer C, Fossion E, Vaillant JM. Sinus aspergillosis. J Craniomaxillofac Surg. 1990; 18: 33-40.

7. Mensi M, Piccioni M, Marsili F, Nicolai P, Sapelli PL, Latronico N. Risk of maxillary fungus ball in patients with endodontic treatment on maxillary teeth: a case-contro study. Oral Surg Oral Med Oral Pathol Oral Radiol Endod. 2007; 103: 433-443.

8. Legent F, Billet J, Beauvillain C, Bonnet J, Miegeville $M$. The role of dental canal fillings in the development of Aspergillus sinusitis. A report of 85 cases. Arch Otorhinol Laryngol. 1989; 246: 318-332.

9. Park GY, Kim HY, Min JY, Dhong HJ, Chung SK. Endodontic treatment: a significant risk factor for the development of maxillary fungal ball. Clin Exp Otorhinolaryngol. 2010; 3 136-140.

10. Berrylin JF. Fungus balls of the paranasal sinuses. Otolaryngol Clin North Am. 2000; 33: 389-398.

11. De Shazo RD, Chapin K, Swain RE. Fungal sinusitis, N Engl J Med. 1997; 337: 254-259.

12. Hora JF. Primary aspergillosis of the paranasal sinuses and associated areas. Laryngoscope. 1965; 75: 768-773.

13. Stammberger $H$, Jakse $R$, Beaufort $F$ Aspergillosis of the paranasal sinuses $\mathrm{x}$-ray diagnosis, histopathology and clinical aspects. Ann Otol Rhinol Laryngol. 1984; 93: 251-225.

14. Uri N, Cohen-Kerem R, Elmalah I, Doweck I, Greenberg E, Classification of fungal sinusitis in immunocompetent patients, Otolaryngol Head Neck Surg. 2003; 129 372-378.

15. Pagella F, Matti E, De Bernardi F, et al. Paranasal sinus fungus ball: diagnosis and management. Mycoses. 2007; 50: 451-456.

16. Klossek JM, Serrano E, Peloquin L, Percodan J, Fontanel JP, Pessey JJ. Functional endoscopic sinus surgery and 109 mycetomas of paranasal sinuses. Laryngoscope. 1997; 107: 112-117

17. De Shazo RD, O'Brien M, Chapin K, et al Criteria for the diagnosis of sinus myce- toma. J Allergy Clin Immunol. 1997; 99: 475485

18. Katzenstein AL, Sale SR, Greenberger PA. Allergic aspergillus sinusitis: a newly recognized form of sinusitis. J Allergy Clin Immunol. 1983; 72: 89-93.

19. de Shazo RD, Swain RE. Diagnostic criteria for allergic fungal sinusitis. J Allergy Clin Immunol. 1995. 96: 24-35.

20. Bartynski JM, McCaffrey TV, Frigas E. Allergic fungal sinusitis secondary to dermatiaceous fungi-Curvularia lunata and Alternaria. Otolaryngol Head Neck Surg. 1990; 103: 32-39.

21. Ponikau JU, Sherris DA, Kern EB, et al. The diagnosis and incidence of allergic fungal sinusitis. Mayo Clin Proc. 1999; 74: 877-884.

22. Braun H, Buzina W, Freudenschuss K, Beham A, Stammberger $\mathrm{H}$. Eosinophilic fungal rhinosinusitis: a common disorder in Europe ? Laryngoscope. 2003; 113: 264-269.

23. Dufour X, Kauffmann-Lacroix C, Ferrie JC, Goujon JM, Rodier MH, Klossek JM. Paranasal sinus fungus ball: epidemiology, clinica features and diagnosis. A restrospective analysis of 173 cases from a single medical center in France, 1989-2002. Med Mycol. 2006; 44: 61-67.

24. Nicolai P, Lombardi D, Tomenzoli D, et al. Fungus ball of the paranasal sinuses : experience in 160 patients treated with endoscopic surgery. Laryngoscope. 2009; 119 2275-2279.

25. Toussain G, Botterel FR, Alsamad IA, et al. Sinus fungus balls: characteristics and management in patients with host factors for invasive infection, Rhinology. 2012, 50: 269276 .

26. Bambule G, Grigoriu D, Savary M, Delacretaz J. [Mycotic rhinosinusitis and its treatment], Ann Otolaryngol Chir Cervicofac. 1982; 99: 531-535.

27. Barry B, Topeza M, Gehanno P. Aspergillosis of the paranasal sinus and environmental factors, Ann Otolaryngol Chir Cervicofac. 2002; 119: 170-173.

28. Lew D, Southwick FS, Montgomery WW, Weber AL, Baker AS, Sphenoid sinusitis: a review of 30 cases. N Engl J Med. 1983, 309: 1149-1154.

29. Hnatuk LA, MacDonald RE, Papsin BC, Isolated sphenoid sinusitis: the Toronto Hospital for Sick Children experience and review of the literature. J Otolaryngol. 1994, 23: $36-41$

30. Castelnuovo P, Pagella F, Semino L, De bernardi F, Delu G. Endoscopic treatment of the isolated sphenoid sinus lesion. Eur Arch Otorhinolaryngol. 2005; 262, 142-147.

31. Lawson W, Reino AJ. Isolated sphenoid sinus disease: an analysis of 132 cases, Laryngoscope. 1997, 107: 1590-1595.

32. Martin TJ, Smith TL, Smith MM, Loehrl TA, Evaluation and surgical management of isolated sphenoid sinus disease. Arch Otolaryngol Head Neck Surg. 2002, 128 :
1413-1419.

33. Socher JA, Cassano M, Filheiro CA, Cassano P, Felippu A. Diagnosis and treatment of isolated sphenoid sinus disease: a review of 109 cases. Acta Otolaryngol. 2008, 128: 1004-1010.

34. Bowman J, Panizza B, Gandhi M. Sphenoid sinus fungal balls. Ann Otol Rhinol Laryngol. 2007; 116: 514-519.

35. Ferreiro JA, Carlson BA, Cody DT, 3rd. Paranasal sinus fungus balls. Head Neck. 1997; 19: 481-486.

36. Leroux E, Valade D, Guichard JP, Herman P. Sphenoid fungus balls: clinical presentation and long-term follow-up in 24 patients. Cephalagia. 2009; 29: 1218-1223.

37. Lee TJ, Huang SF, Chang PH. Characteristics of isolated sphenoid sinus aspergilloma: report of twelve cases and literature review. Ann Otol Rhinol Laryngol. 2009; 118: 211 217.

38. Klossek JM, Peloquin L, Fourcroy PJ, Ferrie JC, Fontanel JP. Aspergillomas of the sphenoid sinus: a series of 10 cases treated by endoscopic sinus surgery. Rhinology. 1996; 34: 179-183.

39. Thery A, Espitalier F, Cassagnau E, Durand $\mathrm{N}$, Malard O. Caractéristiques cliniques et évolutives de l'aspergillose sphénoïdale: étude à partir de 15 cas rétrospectifs, Eur Ann Otorhinolaryngol Head Neck Dis. 2012; 129: 213-218.

40. Yiotakis I, Psarommatis I, Seggas I, Manolopoulos L, Ferekidis E, Adamopoulos G. Isolated sphenoid sinus aspergillomas. Rhinology 1997; 35: 136-139.

41. Cho SH, Jin BJ, Lee YS, Paik SS, Ko MK, Yi HJ. Orbital apex syndrome in a patient with sphenoid fungal balls, Clin Exp Otorhinolaryngol. 2009; 2: 52-54.

42. Pushker N, Meel R, Kashyap S, Bajaj MS, Sen S. Invasive aspergillosis of orbit in immunocompetent patients: treatment and outcome. Ophthalmology. 2011; 118: 18861891.

43. Sivak-Callcott JA, Livesley N, Nugent RA, Rasmussen SL, Saeed P, Rootman J, Localised invasive sino-orbital aspergillosis: characteristic features, $\mathrm{Br} J$ Ophthalmol. 2004; 88: 681-687.

44. Dhong HJ, Jung JY, Park JH. Diagnostic accuracy in sinus fungus balls: CT scan and operative findings. Am J Rhinol. 2000; 14: 227-231.

45. Fatterpekar G, Mukherji S, Arbealez A, Maheshwari S, Castillo M. Fungal diseases of the paranasal sinuses. Semin Ultrasound CT MR.1999; 20: 391-401.

46. Zinreich SJ, Kennedy DW, Malat J, et al. Fungal sinusitis: diagnosis with CT and MR imaging. Radiology. 1988; 169: 439-444.

47. Johns ME, Rosenbaum AE. Fungal sinusitis: diagnosis with CT and MR imaging. Radiology. 1988; 169: 439-444.

48. Schlosser RJ, Gross CW, Kountakis S. Skull base erosion by sphenoid fungus balls: 
diagnosis and endoscopic treatment. Am Rhinol. 2002; 16: 161-164.

49. Row-Jones JM, Moore-Gillon V. Destructive noninvasive paranasal sinus aspergillosis: component of a spectrum of disease. $J$ Otolaryngol. 1994; 23: 92-96.

50. Lee DH, Kim SK, Joo YE, Lim SC. Fungus ball within a mucocele of the sphenoid sinus and infratemporal fossa: case report with radiological findings. J Laryngol Otol. 2012; 126: 210-213.

51. Broglie MA, Tinguely M, Holzmann D. How to diagnose sinus fungus balls in the paranasal sinus? An analysis of an institution's cases from January 1999 to December
2006, Rhinology. 2009; 47: 379-384

52. Chao TK, Liu CM. Gauze-assisted technique in endoscopic removal of fungus ball of the maxillary sinus. Am J Rhinol. 2006; 20 417-420.

\section{Pr Philippe Eloy}

ENT Department

CHU Mont-Godinne

5530 Yvoir

Belgium

Tel: + 32-81-42 3711

Fax: + 32-81-42 3703

E-mail: philippe.eloy@uclouvain.be 